\title{
Einführung in den Themenstrang „Intersektionalität in der Sonderpädagogik“
}

\author{
Carmen Zurbriggen, Winfried Kronig \\ Universität Freiburg/Schweiz
}

\begin{abstract}
Noch weitgehend unbemerkt von der Sonderpädagogik beginnt sich in einigen unserer Nachbardisziplinen ein Denken durchzusetzen, welches soziale Ungleichheitslagen zeitgleich mithilfe mehrerer Analysekategorien zu berücksichtigen sucht. Grundannahme einer solchen intersektionalen Denkweise ist, dass „Menschen anhand einer ganzen Reihe von Differenzmerkmalen wahrgenommen und einer sozialen Bewertung unterzogen werden“ (Dederich 2014, 48). Die verschiedenartigen, sich aus sozialen Strukturen und Praxen generierenden Unterschiede werden dabei nicht additiv, sondern interdependent und in Hinblick auf die aus der Wechselbeziehung resultierenden Ungleichheitskonstellationen untersucht.
\end{abstract}

Selbstverständlich: Kaum jemand aus dem Bereich der Sonderpädagogik würde Behinderung als eine konsistente, in sich geschlossene personale Kategorie bezeichnen. Nicht nur Behinderungen - als Ergebnis von sozialen Zuordnungsprozessen zu ähnlichen Phänomenbereichen differieren. Personen mit einer Behinderung sind unterschiedlich. Es sind Frauen und Männer, Ansässige und Zugezogene, jüngere und ältere Personen, reichere und ärmere. Behinderung steht gewissermaßen im Schnittfeld von sozialen Ungleichheitskategorien und -dimensionen.

Und dennoch steckt die systematische Beschäftigung mit dem Zusammenspiel verschiedener Ungleichheitsdimensionen in der Sonderpädagogik noch in den Anfängen. Überdies nimmt die Kategorie Behinderung bislang in der Intersektionalitätsforschung ein Nischendasein ein (z. B. Baldin 2014). Demgemäß existieren etliche offene Fragen und sich konkurrierende
Auffassungen. So gilt es etwa für die intersektionale Analyse die Besonderheit der Kategorie Behinderung im Vergleich $\mathrm{zu}$ anderen Ungleichheitskategorien deutlicher herauszuarbeiten. Des Weiteren besteht kein Konsens darüber, „welche Differenzkategorien und -linien sich mit Behinderung, dynamisch überkreuzen“" (Lindmeier 2013, 120).

Der nachfolgende Beitrag von Katharina Walgenbach eröffnet nun diesen Themenstrang rund um Intersektionalität und Behinderung und versucht zu ergründen, was „Intersektionalität als Paradigma“ für unser Erkennen dieser nicht klar festzulegenden Personengruppe in der Theoriebildung, in der Forschung sowie in der Praxisgestaltung bedeutet. In den kommenden Ausgaben folgen Fachbeiträge, welche das Konstrukt Behinderung in Interdependenz mit verschiedenen sozialen Ungleichheitskategorien aus der Perspektive von Intersektionalität beleuchten. Gemeinsam bergen sie das Versprechen, das Diskursfeld Behinderung für die Sonderpädagogik wesentlich zu erweitern.

\section{Literatur}

Baldin, D. (2014): Behinderung - eine neue Kategorie für die Intersektionalitätsforschung? In: Wansing, G.; Westphal, M. (Hrsg.): Behinderung und Migration. Inklusion, Diversität, Intersektionalität. Wiesbaden: Springer VS, 49-71

Dederich, M. (2014): Intersektionalität und Behinderung. Ein Problemaufriss. In: Behinderte Menschen $37,47-53$

Lindmeier, C. (2013): Das aktuelle Thema: Intersektionalität. In: Sonderpädagogische Förderung heute $58,119-120$ 